\title{
ARTICLE
}

Epidemiology

\section{Acid-suppressive medications and risk of colorectal cancer: results from three large prospective cohort studies}

\author{
Ana Babic ${ }^{1}$, Xuehong Zhang ${ }^{2}$, Vicente Morales-Oyarvide ${ }^{1}$, Chen Yuan ${ }^{1}$, Natalia Khalaf ${ }^{3}$, Hamed Khalili, ${ }^{4,5}$, Paul Lochhead ${ }^{4}$, \\ Andrew T. Chan ${ }^{2,4,5,6}$, Shuji Ogino ${ }^{1,6,7,8}$, Brian M. Wolpin ${ }^{1}$, Kana Wu ${ }^{9}$, Charles S. Fuchs ${ }^{2,10,11,12}$, Edward L. Giovannucci ${ }^{2,8,9}$, \\ Meir J. Stampfer ${ }^{2,8,9}$ and Kimmie $\mathrm{Ng}^{1}$
}

BACKGROUND: Despite several plausible biological mechanisms linking proton pump inhibitors (PPIs) and $\mathrm{H} 2$ receptor antagonists (H2RAs) with colorectal tumorigenesis, their association with risk of colorectal cancer (CRC) has not been adequately assessed in prospective epidemiological studies.

METHODS: We evaluated the association of acid-suppressive medication use with CRC risk among 175,871 (PPI) and 208,831 (H2RA) participants from three large prospective cohort studies. Medication use was assessed at baseline and updated biennially. The association was evaluated using multivariate Cox proportional hazards regression models.

RESULTS: There was no significant association between baseline PPI use (hazard ratio $(\mathrm{HR})=0.89,95 \%$ confidence interval $(\mathrm{Cl})$, 0.71-1.12) or PPI use after a lag of 8-10 years ( $\mathrm{HR}=1.12,95 \% \mathrm{Cl}, 0.78-1.59)$ with $\mathrm{CRC}$ risk. We observed no significant association between $\mathrm{H} 2 \mathrm{RA}$ use after a lag of 8-10 years and $\mathrm{CRC}$ risk $(\mathrm{HR}=1.02,95 \% \mathrm{Cl}, 0.81-1.28)$, while risk was lower for participants with baseline $\mathrm{H} 2 \mathrm{RA}$ use $(\mathrm{HR}=0.76,95 \% \mathrm{Cl}, 0.60-0.95)$. Duration of PPI use or H2RA use was not associated with $\mathrm{CRC}$ risk $(P$-trend $=0.21$ and 0.95 , respectively).

CONCLUSIONS: Among participants from three large prospective cohorts, use of PPI or H2RA was not associated with higher risk of colorectal cancer.

British Journal of Cancer (2020) 123:844-851; https://doi.org/10.1038/s41416-020-0939-y

\section{BACKGROUND}

Acid-suppressive medications, including proton pump inhibitors (PPIs) and histamine type 2 receptor antagonists (H2RAs), are among the most widely used medications in the United States. ${ }^{1}$ These are indicated for the treatment of upper gastrointestinal acid-related disorders, including gastrointestinal reflux disease and peptic ulcer disease, ${ }^{2}$ but lack of adherence to prescription guidelines, as well as over-the-counter availability, have led to their increasing overuse. ${ }^{3}$

Although PPI and H2RA are generally considered safe, ${ }^{4}$ concerns have been raised about long-term use and risk of colorectal cancer. PPI- and H2RA-induced suppression of gastric acid leads to elevated systemic levels of the hormone gastrin, ${ }^{5-7}$ which induces proliferation of colorectal epithelium and colon adenoma progression in animal models. ${ }^{8-10}$ Elevated gastrin levels have been associated with increased colorectal cancer risk in epidemiologic studies. ${ }^{11}$ Second, several recent studies revealed large differences in microbiota composition between PPI users and non-users. ${ }^{12-14}$ Although the relationship between colorectal cancer and the gut microbiota is not yet well understood, several studies strongly implicate specific bacterial species in the development of colorectal tumours. ${ }^{15,16}$ Furthermore, Fusobacterium nucleatum, a Gram-negative anaerobic commensal associated with increased colorectal cancer risk, ${ }^{17,18}$ shows in vitro sensitivity to lansoprazole and omeprazole, the two most commonly used PPIs. ${ }^{19,20}$

Despite a potential biological link between acid-suppressive medications and colorectal cancer, this relationship has not been sufficiently investigated in epidemiological studies. Case-control studies suggested increased risk ${ }^{21,22}$ or no association ${ }^{23-26}$ with $\mathrm{PPI}$ use, and no association with H2RA use, ${ }^{21}$ but prospective cohort studies are sparse. ${ }^{27}$ Furthermore, previous studies had important limitations, including inadequate adjusting for potential confounders, such as history of colonoscopy and BMI, small number of cases and a relatively short duration of follow-up. Most importantly, since colorectal cancer has a long induction period of at least 10 years, ${ }^{28}$ it is crucial to carefully examine timing of exposure in relation to cancer diagnosis. We therefore conducted

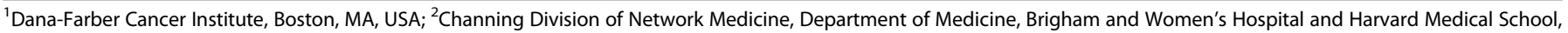

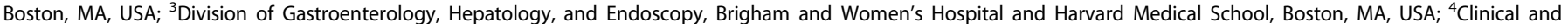

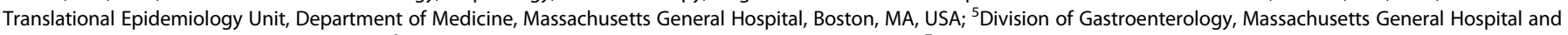

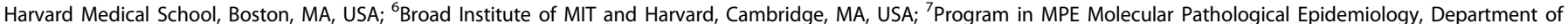

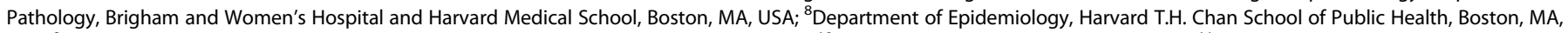

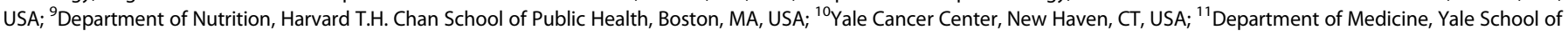
Medicine, New Haven, CT, USA and ${ }^{12}$ Smilow Cancer Hospital, New Haven, CT, USA
} Correspondence: Ana Babic (ababic1@partners.org)

Received: 7 January 2020 Revised: 5 May 2020 Accepted: 28 May 2020 Published online: 16 June 2020 
a prospective analysis to evaluate the association between use of acid-suppressive medications and colorectal cancer risk within three large cohorts (Nurses' Health Study [NHS], Nurses' Health Study II [NHSII] and Health Professionals Follow-Up Study [HPFS]) with updated information on PPI and H2RA use, with up to 26 years of follow-up.

\section{METHODS}

Study population

The NHS, NHSII and HPFS are ongoing US-based prospective cohort studies. The NHS was established in 1976 among 121,700 US female registered nurses aged $30-55$ years, ${ }^{29,30}$ and NHSII was established in 1989 among 116,429 US female nurses aged 25-42 years. ${ }^{31}$ HPFS was established in 1986 among 51,529 US male health professionals aged $40-75$ years at baseline. ${ }^{32}$ In all three cohorts, participants completed an initial questionnaire about their medical history, lifestyle factors and health behaviours. Participants have been mailed follow-up questionnaires every 2 years to provide updated information on those factors, as well as disease outcomes. Participants were also asked to complete semiquantitative validated food frequency questionnaires (FFQs) approximately every 4 years. Follow-up is $>90 \%$ for all three cohorts. The study protocol was approved by the institutional review boards of the Brigham and Women's Hospital and Harvard T.H. Chan School of Public Health, and those of participating registries as required.

The eligible population for the analysis of PPI use consisted of participants who answered the questionnaire in which PPI use was first assessed (NHS, 2000; NHSII, 2001; HPFS, 2004). We excluded participants with previous history of cancer, except nonmelanoma skin cancer and history of ulcerative colitis or Crohn's disease. After those exclusions, a total of 175,871 participants were included in the analysis. Among eligible participants who answered the first questionnaire where H2RA use was assessed (NHS, 1994; NHSIl, 1995; HPFS, 1988), we applied the same exclusions, leaving a total of 208,831 participants.

\section{Ascertainment of PPI and H2RA use}

Information about PPI use and H2RA use was obtained from the baseline questionnaire, and updated in each subsequent biennial cycle. At baseline, participants were asked about regular use (yes/ no) of Prilosec or Prevacid in NHS and NHSII, and Prilosec, Nexium, Prevacid (lansoprazole), Protonix or Aciphex in HPFS in the past 2 years. Similarly, they were asked about regular use of ranitidine (Zantac) or cimetidine (Tagamet) in NHS, cimetidine (Tagamet) or other $\mathrm{H} 2$ blockers (e.g. Zantac, Pepcid, Axid) in NHSII, and cimetidine or ranitidine (e.g. Tagamet, Zantac) in HPFS in the past 2 years. If a participant's information on medication use was missing for a follow-up cycle, then that participant did not contribute person-time to the analysis for that cycle.

\section{Ascertainment of covariates}

Information about demographic and lifestyle factors known to be associated with colorectal cancer was obtained from the baseline and follow-up questionnaires. These included age, height, weight, physical activity, family history of colorectal cancer, regular use of aspirin and non-aspirin nonsteroidal anti-inflammatory drugs (NSAIDs), menopausal status, menopausal hormone therapy (MHT) use, multivitamin use and history of lower endoscopy (including indication for the procedure). All the covariates were updated biennially, except for family history of colorectal cancer, which was updated approximately every 4 years. Once a participant reported an endoscopy, positive history of endoscopy was carried forward in all the subsequent cycles. Information on dietary factors, including intake of fibre, vitamin D, calcium, folate, red meat, processed meat, total fat intake and total caloric intake, was extracted from FFQs every 4 years. We used energy-adjusted values and cumulative updated intake, defined as the average intake from all available FFQs up to the most recent biennial follow-up questionnaire. Participants were asked about a history of gastric or duodenal ulcer in NHS only at two time points (2000, 2008); in the other two cohorts, this information was updated every 2 years after the baseline (1991 in NHSII, 1986 in HPFS).

Documentation of colorectal cancer cases and deaths Information about disease outcomes, including colorectal cancer, was obtained in biennial questionnaires. For each participant reporting a colorectal cancer diagnosis, we asked for permission to obtain medical records and pathology reports. Deaths of participants were identified through family members, National Death Index or the US postal service. If the review of death certificate revealed colorectal cancer as the cause of death, we sought permission from next of kin to obtain and review medical records. A study physician blinded to exposure status reviewed medical records, and extracted information on cancer stage, histopathologic features and tumour location.

\section{Statistical analysis}

Due to the similar questionnaire design across cohorts, we combined data from all three cohorts into a single dataset. Person-time was calculated as time from the return of baseline questionnaires until colorectal cancer diagnosis, diagnosis of any cancer, except non-melanoma skin cancer, death or the end of follow-up (NHS: June 2014; NHSII: June 2015; HPFS: January 2014), whichever came first.

To consider different intervals between exposures and outcome, we used several approaches to modelling exposures, including current, baseline, persistent, cumulative and lagged use of PPI and H2RA. Current use was defined as regular medication use reported in the most recent questionnaire; this variable was updated in each biennial follow-up cycle. Baseline use was assessed on the first questionnaire that asked about PPI or H2RA use, and subsequently carried forward. Persistent use was defined as use reported in the baseline questionnaire plus one or two subsequent follow-up questionnaire cycles. To evaluate the cumulative effect of drug use on cancer risk, we assessed the number of cycles with reported drug use (no use, use in 1-2 cycles, use in $\geq 3$ cycles), regardless of whether these cycles were consecutive or not. Lastly, since the association between several established risk factors and colorectal cancer is observed only after a latency of 10 years or longer, ${ }^{33}$ we examined medication use lagged for varying periods of time up to 10 years. For example, in NHS, PPI use was first assessed in 2000. For a latency of 2-4 years, we used PPI use in 2000 for the follow-up period from 2002 to 2004, PPI use in 2002 for the follow-up period from 2004 to 2006 and so forth. For a latency of 4-6 years, we used PPI use in 2000 for the follow-up period from 2004 to 2006, PPI use in 2002 for the follow-up period from 2006 to 2008 and so on.

We used Cox proportional hazards regression models stratified by age (months) and 2-year questionnaire cycle to estimate the hazard ratios (HRs) and 95\% confidence intervals (Cls) of cancer risk for users of acid-suppressive medications, compared to nonusers. In the main multivariate model, we adjusted for study cohort (NHS, NHSII, HPFS) and established colorectal cancer risk factors, including BMI $\left(<25 \mathrm{~kg} / \mathrm{m}^{2}, 25-27.5 \mathrm{~kg} / \mathrm{m}^{2}, 27.5-30 \mathrm{~kg} / \mathrm{m}^{2}\right.$, $\left.\geq 30 \mathrm{~kg} / \mathrm{m}^{2}\right)$, physical activity $(<3$ metabolic equivalent [MET]-h/ week, 3-27 MET-h/week, $\geq 27$ MET-h/week), family history of colorectal cancer (no, yes), daily caloric intake (quintiles), alcohol intake $(<5 \mathrm{~g} /$ day, $5-15 \mathrm{~g} /$ day, $\geq 15 \mathrm{~g} /$ day $)$, pack-years of smoking $(0,1-10, \geq 10$ pack-years), history of lower endoscopy (never, ever), dietary and supplemental vitamin D intake (quintiles), dietary and supplemental calcium intake (quintiles), regular aspirin use (no, yes), dietary and supplemental folate intake (quintiles), MHT use (premenopausal, postmenopausal never MHT user, postmenopausal past MHT user, postmenopausal current MHT user) (NHS and 
Table 1. Baseline characteristics of participants by use of proton pump inhibitors.

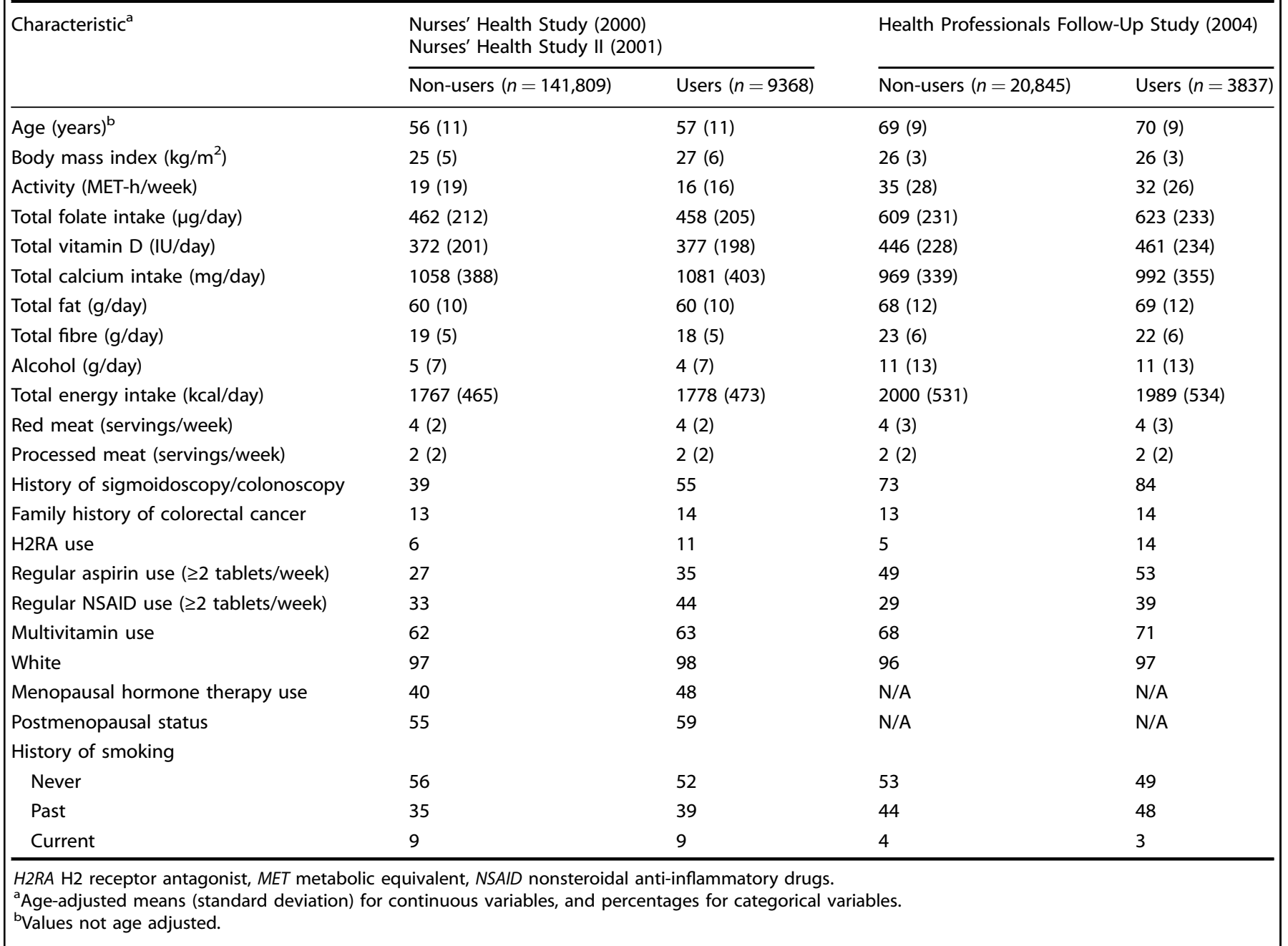

NHSII only) and red meat intake (quintiles). We additionally examined confounding by waist circumference (continuous), waist-to-hip ratio (continuous), regular NSAID use (no, yes) and dietary intake of processed meat (quintiles). To evaluate the heterogeneity of the association by cohort, we pooled the cohortspecific estimates, and calculated the $Q$-statistic using randomeffects meta-analysis. ${ }^{34}$ We performed stratified analyses by gender, history of lower endoscopy (never, ever), BMI $(<25 \mathrm{~kg} /$ $\mathrm{m}^{2}, 25-30 \mathrm{~kg} / \mathrm{m}^{2}, \geq 30 \mathrm{~kg} / \mathrm{m}^{2}$ ) and history of smoking (never, ever) to identify effect modification. We included a cross-product between PPI or H2RA use and stratifying variable in the multivariate model, and tested the significance of interaction using the Wald test. All $P$ values were two-sided, using a 0.05 significance level. All the analyses were performed using the SAS software (SAS Institute, Inc., Version 9.4).

\section{RESULTS}

We documented 1255 colorectal cancer cases during 2.1 million years of follow-up in the PPI use analysis, and 2413 cases during 3.8 million years of follow-up in the H2RA use analysis.

Compared to their respective non-users, both PPI and H2RA users had on average a higher BMI, lower levels of physical activity and higher total calcium intake. Users were more likely to have a history of lower endoscopy, in particular due to gastrointestinal symptoms, and use aspirin, NSAID and multivitamins (Tables 1 and
2). Among women, $\mathrm{PPI}$ and H2RA users were more likely to be postmenopausal and MHT users. There were fewer never smokers among both PPI and H2RA users.

In multivariate adjusted models, there was no significant association between baseline use of PPI and risk of colorectal cancer $(\mathrm{HR}=0.89,95 \% \mathrm{Cl}, 0.71-1.12)$, while current PPI use was associated with decreased risk $(\mathrm{HR}=0.82,95 \% \mathrm{Cl}, 0.68-0.98)$ (Table 3). Persistent PPI use defined as use in the baseline, and one $(\mathrm{HR}=0.83,95 \% \mathrm{Cl}, 0.58-1.18)$ or two subsequent cycles $(\mathrm{HR}=$ $0.84,95 \% \mathrm{Cl}, 0.56-1.24)$ was not significantly associated with risk (Table 3). We next evaluated cumulative PPI use, and observed no significant association between number of cycles with reported PPI use and risk of colorectal cancer $(P$-trend $=0.21)$ (Table 4). To examine different latency periods between PPI use and colorectal cancer diagnosis, we lagged PPI use for up to 10 years, and observed no significant association (8-10 years lag: $H R=1.12$, 95\% Cl, 0.78-1.59) (Table 5).

Use of H2RA at baseline was associated with decreased risk of colorectal cancer $(\mathrm{HR}=0.76,95 \% \mathrm{Cl}, 0.60-0.95)$, while there was no significant association between current or persistent H2RA use (Table 3). Duration of H2RA use was also not significantly associated with risk ( $P$-trend $=0.95$ ) (Table 4$)$. We next examined several latency periods up to 10 years, and observed no significant association (8-10 years lag: $\mathrm{HR}=1.02,95 \% \mathrm{Cl}, 0.81-1.28$ ) (Table 5).

The associations between PPI use and H2RA use and colorectal cancer risk each remained materially unchanged after adjusting 
Table 2. Baseline characteristics of participants by use of $\mathrm{H} 2$ receptor antagonists.

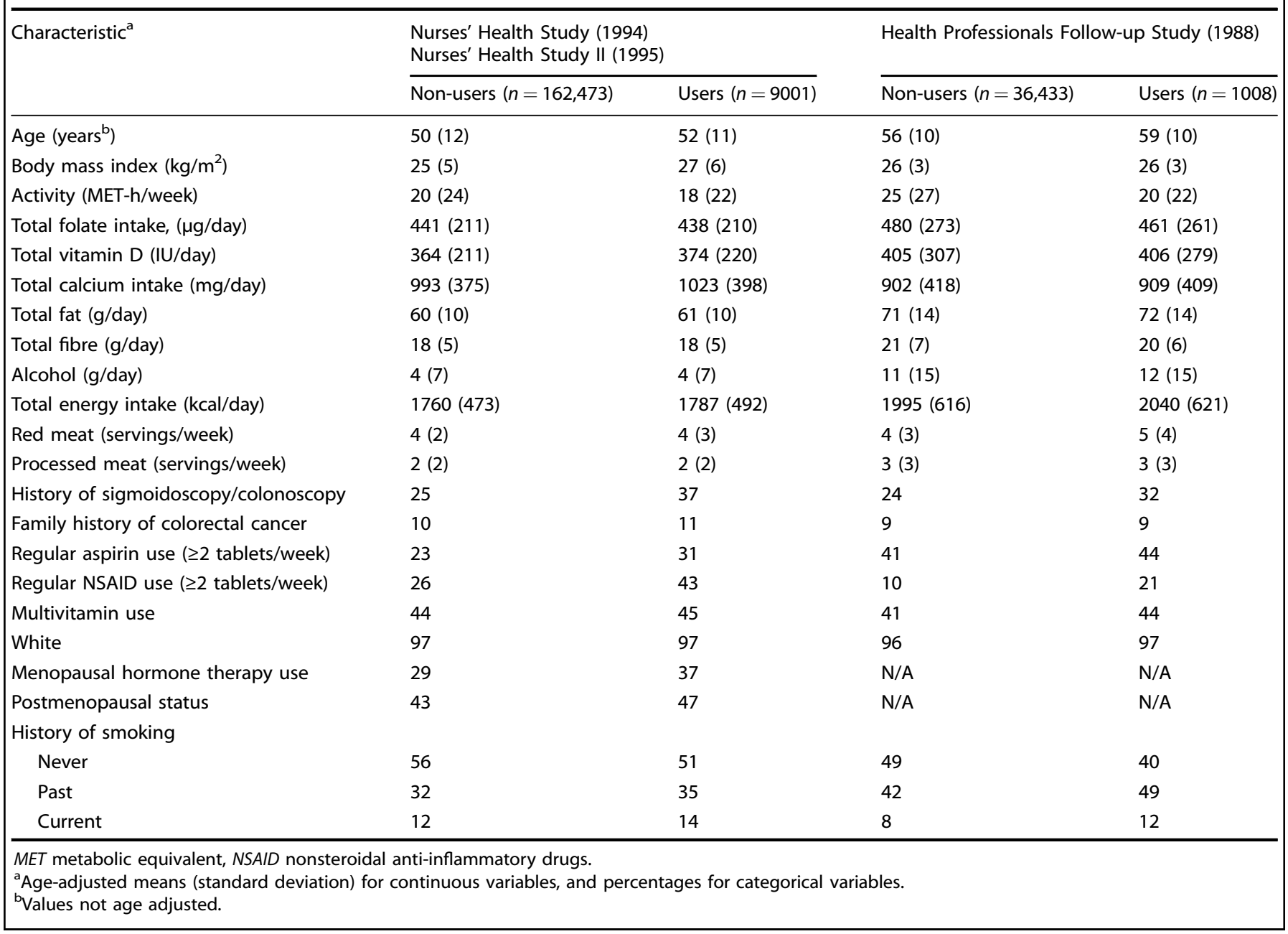

for additional potential confounders, including waist circumference, waist-to-hip ratio, NSAID use, intake of processed meat and history of gastric or duodenal ulcer (data not shown). Associations between both medications and risk of colorectal cancer were similar by primary tumour location (Supplementary Table 1) and by study cohort ( $P$-heterogeneity $\geq 0.13$ ). Furthermore, the associations for both medications and colorectal cancer risk were similar in analyses stratified by sex, history of lower endoscopy, $\mathrm{BMI}$ and smoking status (all $P$-interaction $\geq 0.24$ ) (Supplementary Table 2). Since PPI users were also more likely to use H2RA (Table 1), we adjusted simultaneously for use of both medications to examine their independent association with colorectal cancer. Neither baseline use of PPI $(\mathrm{HR}=0.95,95 \% \mathrm{Cl}, 0.75-1.21)$ nor $\mathrm{H} 2 \mathrm{RA}(\mathrm{HR}=0.82,95 \% \mathrm{Cl}, 0.59-1.12)$ was associated with risk of colorectal cancer in these models.

\section{DISCUSSION}

In this pooled analysis of three large prospective US cohorts, we found no evidence of overall significant association between use of PPI or H2RA medications and risk of colorectal cancer.

Use of both PPI and H2RA was examined using several approaches, with varying latency periods between medication use and colorectal cancer diagnosis. In the analysis of PPI use, we observed no significant association with baseline, persistent, cumulative or up to 10-year lagged medication use and colorectal cancer risk. We observed decreased colorectal cancer risk in current users of PPI; however, use in the past 2 years before diagnosis is not biologically likely to play a role in disease development, and is more likely to be affected by subclinical disease. There was no significant change in colorectal cancer risk in current H2RA users. We did observe a $23 \%$ decrease in colorectal cancer risk that was statistically significant among baseline H2RA users. However, we observed no association with persistent use or longer duration use, arguing against the inverse association between H2RA and colorectal cancer being causal. Overall, these observations do not support the causal role of PPI or H2RA in colorectal cancer aetiology.

Several case-control studies have examined PPI use in relation to colorectal cancer risk, and reported either no association ${ }^{23-26}$ or moderately increased risk among PPI users. ${ }^{21,22}$ One case-control study reported no association between H2RA use and risk of colorectal cancer. ${ }^{21}$ While most of those studies relied on information about medication use extracted from health insurance records or medical charts; therefore, eliminating recall bias, over-the-counter use of these medications was not considered, which could lead to exposure misclassification. Furthermore, several studies failed to account for important confounders such as BMI or history of colonoscopy. ${ }^{21-25}$ In our study, users of PPI and H2RA had a higher BMI and were more likely to have a history of colonoscopy, which have been associated with increased or decreased risk of $\mathrm{CRC}$, respectively. ${ }^{35,36}$ Most importantly, in previous studies timing of medication use in relation to colorectal cancer diagnosis was not carefully examined. Colorectal cancer 
Table 3. Association between use of acid-suppressive medications and risk of colorectal cancer.

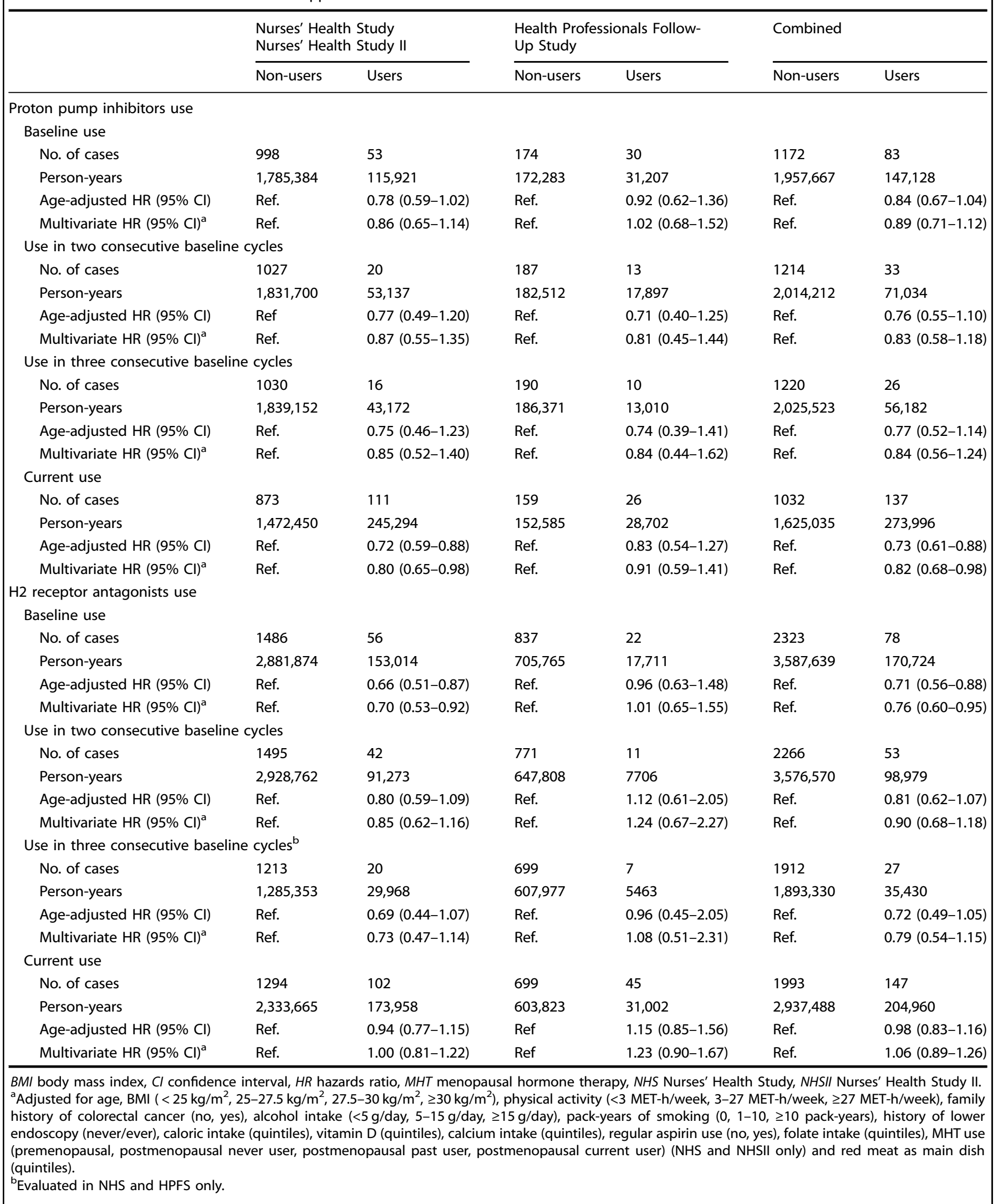


Table 4. Duration of acid-suppressive medications use and risk of colorectal cancer.

\begin{tabular}{|c|c|c|c|c|}
\hline & No use & Used in 1-2 cycles & Used in $\geq 3$ cycles & $P$-trend \\
\hline No. of cases & 952 & 159 & 58 & \\
\hline Age-adjusted HR (95\% Cl) & Ref. & $0.80(0.68-0.95)$ & $0.79(0.60-1.04)$ & 0.01 \\
\hline Multivariate $\mathrm{HR}(95 \% \mathrm{Cl})^{\mathrm{a}}$ & Ref. & $0.89(0.75-1.06)$ & $0.92(0.69-1.21)$ & 0.21 \\
\hline Person-years & $2,652,702$ & 378,358 & 111,387 & \\
\hline Age-adjusted HR (95\% Cl) & Ref. & $0.84(0.73-0.97)$ & $1.02(0.82-1.25)$ & 0.33 \\
\hline Multivariate $\mathrm{HR}(95 \% \mathrm{Cl})^{\mathrm{a}}$ & Ref. & $0.91(0.79-1.05)$ & $1.09(0.88-1.35)$ & 0.95 \\
\hline \multicolumn{5}{|c|}{$\begin{array}{l}\text { BMI body mass index, } \mathrm{Cl} \text { confidence interval, } H R \text { hazards ratio, MHT menopausal hormone therapy, NHS Nurses' Health Study, NHSII Nurses' Health Study II. } \\
\text { aAdjusted for age, BMI }\left(<25 \mathrm{~kg} / \mathrm{m}^{2}, 25-27.5 \mathrm{~kg} / \mathrm{m}^{2}, 27.5-30 \mathrm{~kg} / \mathrm{m}^{2}, \geq 30 \mathrm{~kg} / \mathrm{m}^{2}\right) \text { physical activity }(<3 \mathrm{MET}-\mathrm{h} / \text { week, } 3-27 \mathrm{MET}-\mathrm{h} / \text { week, } \geq 27 \mathrm{MET}-\mathrm{h} / \text { week), family } \\
\text { history of colorectal cancer (no, yes), alcohol intake }(<5 \mathrm{~g} / \mathrm{day}, 5-15 \mathrm{~g} / \mathrm{day}, \geq 15 \mathrm{~g} / \text { day), pack-years of smoking }(0,1-10, \geq 10 \mathrm{pack}-\mathrm{years}), \text { history of lower } \\
\text { endoscopy (never/ever), caloric intake (quintiles), vitamin } \mathrm{D} \text { (quintiles), calcium intake (quintiles), regular aspirin use (no, yes), folate intake (quintiles), MHT use } \\
\text { (premenopausal, postmenopausal never user, postmenopausal past user, postmenopausal current user) (NHS and NHSIl only) and red meat as main dish } \\
\text { (quintiles). }\end{array}$} \\
\hline
\end{tabular}

Table 5. Association between colorectal cancer and lagged use of acid-suppressive medications.

\begin{tabular}{|c|c|c|c|c|c|c|c|c|}
\hline No. of cases & 831 & 108 & 662 & 84 & 496 & 47 & 331 & 36 \\
\hline Person-years & $1,373,645$ & 219,447 & $1,118,996$ & 163,824 & 877,318 & 115,076 & 631,274 & 69,835 \\
\hline $\begin{array}{l}\text { Age-adjusted HR } \\
(95 \% \mathrm{Cl})\end{array}$ & Ref. & $\begin{array}{l}0.76(0.62 \\
\text { to } 0.94)\end{array}$ & Ref. & $\begin{array}{l}0.81(0.65 \\
\text { to } 1.03)\end{array}$ & Ref. & $\begin{array}{l}0.72(0.53 \\
\text { to } 0.97)\end{array}$ & Ref. & $\begin{array}{l}1.01(0.71 \\
\text { to } 1.44)\end{array}$ \\
\hline \multicolumn{9}{|l|}{ H2RA use } \\
\hline No. of cases & 1857 & 126 & 1650 & 99 & 1430 & 88 & 1180 & 78 \\
\hline Person-years & $2,615,912$ & 179,320 & $2,274,028$ & 153,533 & $1,954,486$ & 131,335 & $1,639,395$ & 109,876 \\
\hline $\begin{array}{l}\text { Age-adjusted HR } \\
(95 \% \mathrm{Cl})\end{array}$ & Ref. & $0.92(0.76-1.10)$ & Ref. & $0.82(0.67-1.01)$ & Ref. & $0.87(0.70-1.08)$ & Ref. & $0.96(0.76-1.21)$ \\
\hline
\end{tabular}

has a long latency period of 10 or more years, and a long latency period was required to observe the protective effect of aspirin in prospective cohort studies. ${ }^{37}$ We considered several exposure windows, and observed no association with risk of colorectal cancer with medication use lagged up to 10 years. Furthermore, overall duration of follow-up in our study was up to 26 years, compared with up to 15 years in previous studies, ${ }^{26}$ additionally increasing our ability to detect any potential association.

To our knowledge, this is the first prospective cohort study to evaluate H2RA use in relation to risk of colorectal cancer. One previous prospective cohort study also found no overall increase in colorectal cancer risk in PPI users; ${ }^{27}$ however, this study had unclear time interval between exposure assessment and beginning of follow-up, lacked information on exposure and covariate updating, and did not consider some important confounders, such as history of colonoscopy. We were able to adjust for multiple confounders and examine multiple classifications of updated medication use, and we found no evidence of significant association between PPI use and risk of colorectal cancer.

Both PPI and H2RA lead to increased circulating levels of gastrin, ${ }^{5-7}$ which has been associated with increased risk of colorectal cancer in prospective studies. ${ }^{11}$ In animal models of colorectal cancer, gastrin leads to increased proliferation of colon mucosa and adenoma progression. ${ }^{8-10}$ It is possible that the rise in gastrin levels due to PPI and H2RA use is not sufficient to promote development of colorectal tumours. Higher doses of medication over long periods of time may be necessary to exert an effect on tumour progression. While information on medication dosing was not available in our study, we observed no association with longer duration of use of acid-reducing medications with 
colorectal cancer. We also observed no increase in risk among patients with consistent PPI and H2RA use.

Furthermore, use of acid-suppressive medications leads to inhibition of gastric acid secretion and increased gastric $\mathrm{pH}$, altering both the gastric microbiome and gut microbiome. ${ }^{38}$ Recent studies have revealed significant differences in the composition of the gut microbiome between PPI users and nonusers, ${ }^{12-14}$ and growing evidence suggests a role of an altered microbiome in colorectal cancer development. ${ }^{15,16}$ However, it is not clear whether microbiome changes related to PPI use are overlapping with those associated with colorectal cancer. Our results do not suggest an adverse effect of microbiome changes caused by PPI use on colorectal cancer development.

A strength of this study is the prospective design that avoids the recall bias that often occurs in case-control studies, the large number of colorectal cancer cases and long follow-up in three independent cohorts of men and women. We collected information on the use of two acid-suppressive medications with different mechanisms of action; this use was updated every 2 years. Due to extensive information about lifestyle and dietary factors, we were able to adjust for important variables associated with both medication use and colorectal cancer, thereby minimising potential confounding. We were also able to evaluate confounding by indication, by accounting in our analyses for peptic or gastric ulcers, common reasons for use of acid-suppressive medications.

This study has several limitations. Since the questionnaire asked about "regular use" of the acid-suppressive medications in the previous 2-year cycle, without specifying the dose or frequency, we were not able to investigate a more precise dose-response relationship between acid-suppressive medication use and colorectal cancer. We had limited power to examine in more detail the associations with different tumour locations. Furthermore, evaluation of an even longer latency period might be necessary to further confirm the observed lack of association. Most of the participants in our cohort study were white, and our observations may therefore not be generalisable to other races and/or ethnicities.

In conclusion, in this prospective analysis among participants of three large cohorts, we observed no association between use of acid-suppressive medications and risk of colorectal cancer. Given the highly prevalent use of acid-suppressive medications in the US population, the lack of an association with increased colorectal cancer risk is reassuring.

\section{ACKNOWLEDGEMENTS}

We would like to thank the following state cancer registries for their help: $A L, A Z, A R$, $C A, C O, C T, D E, F L, G A, I D, I L, I N, I A, K Y, L A, M E, M D, M A, M I, N E, N H, N J, N Y, N C, N D$, $\mathrm{OH}, \mathrm{OK}, \mathrm{OR}, \mathrm{PA}, \mathrm{RI}, \mathrm{SC}, \mathrm{TN}, \mathrm{TX}, \mathrm{VA}, \mathrm{WA}$ and WY. The authors assume full responsibility for analyses and interpretation of these data.

\section{AUTHOR CONTRIBUTIONS}

Designed the study: A.B., K.N. Analysed the data: A.B., X.Z., K.N. Interpreted results: A. B., X.Z., V.M.-O., C.Y., N.K., H.K., P.L., A.T.C., S.O., B.M.W., K.W., C.S.F., E.L.G., M.J.S., K.N. Approved the final manuscript version: A.B., X.Z., V.M.-O., C.Y., N.K., H.K., P.L., A.T.C., S. O., B.M.W., K.W., C.S.F., E.L.G., M.J.S., K.N.

\section{ADDITIONAL INFORMATION}

Ethics approval and consent to participate This study was approved by the Institutional Review Boards of the Brigham and Women's Hospital and Harvard T.H. Chan School of Public Health, and those of participating registries as required. Completion of the baseline questionnaire was considered to imply informed consent.

Data availability Information on obtaining access to NHS, NHSIl and HPFS data is available at https://www.nurseshealthstudy.org/researchers (email: nhsaccess@channing. harvard.edu) and https://sites.sph.harvard.edu/hpfs/for-collaborators.

Competing interests The authors declare no competing interests.
Funding information This work was supported by following research grants from the National Institutes of Health (NIH): UM1 CA167552, UO1 CA176726, UO1 CA186107, P01 CA55075 and P01 CA87969. Additional support from NIH K07 CA222159 to A.B.; NIH K07 CA188126 and American Cancer Society Research Scholar Grant (RSG130476) to X.Z.; NIH R01 CA151993 and R35 CA197735 to S.O.; NIH R01 CA118553 to C.S.F.; Cancer Research UK Grand Challenge OPTIMISTICC Project award to S.O. and K.N.; Crohn's and Colitis Foundation to P.L., NIH R01 CA205406, DOD CA160344 and Project P Fund to K.N. The content is solely the responsibility of the authors and does not necessarily represent the official views of the National Institutes of Health. The funding sources played no role in study design, data collection, data analysis, interpretations of results or manuscript preparation.

Supplementary information is available for this paper at https://doi.org/10.1038/ s41416-020-0939-y.

Note This work is published under the standard license to publish agreement. After 12 months the work will become freely available and the license terms will switch to a Creative Commons Attribution 4.0 International (CC BY 4.0).

Publisher's note Springer Nature remains neutral with regard to jurisdictional claims in published maps and institutional affiliations.

\section{REFERENCES}

1. Center for Disease Control and Prevention. Selected Prescription Drug Classes Used in the Past 30 Days, By Sex and Age: United States, Selected Years 1988-1994 through 2011-2014 (2016).

2. Schubert, M. L. \& Peura, D. A. Control of gastric acid secretion in health and disease. Gastroenterology 134, 1842-1860 (2008).

3. Savarino, V., Dulbecco, P., de Bortoli, N., Ottonello, A. \& Savarino, E. The appropriate use of proton pump inhibitors (PPIs): need for a reappraisal. Eur. J. Intern. Med. 37, 19-24 (2017).

4. Johnson, D. A., Katz, P. O., Armstrong, D., Cohen, H., Delaney, B. C., Howden, C. W. et al. The safety of appropriate use of over-the-counter proton pump inhibitors: an evidence-based review and Delphi consensus. Drugs 77, 547-561 (2017).

5. Festen, H. P., Thijs, J. C., Lamers, C. B., Jansen, J. M., Pals, G., Frants, R. R. et al. Effect of oral omeprazole on serum gastrin and serum pepsinogen I levels. Gastroenterology 87, 1030-1034 (1984).

6. Lazzaroni, M., Sangaletti, O. \& Bianchi Porro, G. Gastric acid secretion and plasma gastrin during short-term treatment with omeprazole and ranitidine in duodenal ulcer patients. Hepato-Gastroenterology 39, 366-370 (1992).

7. Ohsawa, T., Hirata, W. \& Higichi, S. Effects of three H2-receptor antagonists (cimetidine, famotidine, ranitidine) on serum gastrin level. Int. J. Clin. Pharm. Res. 22, 29-35 (2002).

8. Koh, T. J., Dockray, G. J., Varro, A., Cahill, R. J., Dangler, C. A., Fox, J. G. et al. Overexpression of glycine-extended gastrin in transgenic mice results in increased colonic proliferation. J. Clin. Invest. 103, 1119-1126 (1999).

9. Watson, S. A., Morris, T. M., McWilliams, D. F., Harris, J., Evans, S., Smith, A. et al. Potential role of endocrine gastrin in the colonic adenoma carcinoma sequence. Br. J. Cancer 87, 567-573 (2002).

10. Watson, S. A. \& Smith, A. M. Hypergastrinemia promotes adenoma progression in the APC(Min-/+) mouse model of familial adenomatous polyposis. Cancer Res. 61, 625-631 (2001).

11. Thorburn, C. M., Friedman, G. D., Dickinson, C. J., Vogelman, J. H., Orentreich, N. \& Parsonnet, J. Gastrin and colorectal cancer: a prospective study. Gastroenterology 115, 275-280 (1998).

12. Freedberg, D. E., Lebwohl, B. \& Abrams, J. A. The impact of proton pump inhibitors on the human gastrointestinal microbiome. Clin. Lab. Med. 34, 771-785 (2014).

13. Imhann, F., Bonder, M. J., Vich Vila, A., Fu, J., Mujagic, Z., Vork, L. et al. Proton pump inhibitors affect the gut microbiome. Gut 65, 740-748 (2016).

14. Jackson, M. A., Goodrich, J. K., Maxan, M. E., Freedberg, D. E., Abrams, J. A., Poole, A. C. et al. Proton pump inhibitors alter the composition of the gut microbiota. Gut 65, 749-756 (2016).

15. Louis, P., Hold, G. L. \& Flint, H. J. The gut microbiota, bacterial metabolites and colorectal cancer. Nat. Rev. Microbiol. 12, 661-672 (2014).

16. Sears, C. L. \& Garrett, W. S. Microbes, microbiota, and colon cancer. Cell Host Microbe 15, 317-328 (2014).

17. Kostic, A. D., Gevers, D., Pedamallu, C. S., Michaud, M., Duke, F., Earl, A. M. et al. Genomic analysis identifies association of Fusobacterium with colorectal carcinoma. Genome Res 22, 292-298 (2012).

18. Mima, K., Sukawa, Y., Nishihara, R., Qian, Z. R., Yamauchi, M., Inamura, K. et al. Fusobacterium nucleatum and T cells in colorectal carcinoma. JAMA Oncol. 1, 653-661 (2015). 
19. Nguyen, P. T., Baldeck, J. D., Olsson, J. \& Marquis, R. E. Antimicrobial actions of benzimidazoles against oral streptococci. Oral Microbiol. Immunol. 20, 93-100 (2005).

20. Sheng, J., Nguyen, P. T., Baldeck, J. D., Olsson, J. \& Marquis, R. E. Antimicrobial actions of benzimidazoles against the oral anaerobes Fusobacterium nucleatum and Prevotella intermedia. Arch. Oral Biol. 51, 1015-1023 (2006).

21. Chubak, J., Boudreau, D. M., Rulyak, S. J. \& Mandelson, M. T. Colorectal cancer risk in relation to use of acid suppressive medications. Pharmacoepidemiol. Drug Saf. 18, 540-544 (2009)

22. Lai, S. W., Liao, K. F., Lai, H. C., Lin, C. L. \& Sung, F. C. Use of proton pump inhibitors correlates with increased risk of colorectal cancer in Taiwan. Asia Pac. J. Clin. Oncol. 9, 192-193 (2013).

23. Robertson, D. J., Larsson, H., Friis, S., Pedersen, L., Baron, J. A. \& Sorensen, H. T. Proton pump inhibitor use and risk of colorectal cancer: a population-based, case-control study. Gastroenterology 133, 755-760 (2007).

24. Siersema, P. D., Yu, S., Sahbaie, P., Steyerberg, E. W., Simpson, P. W., Kuipers, E. J. et al. Colorectal neoplasia in veterans is associated with Barrett's esophagus but not with proton-pump inhibitor or aspirin/NSAID use. Gastrointest. Endosc. 63 581-586 (2006)

25. van Soest, E. M., van Rossum, L. G., Dieleman, J. P., van Oijen, M. G., Siersema, P. D., Sturkenboom, M. C. et al. Proton pump inhibitors and the risk of colorectal cancer. Am. J. Gastroenterol. 103, 966-973 (2008).

26. Yang, Y. X., Hennessy, S., Propert, K., Hwang, W. T., Sedarat, A. \& Lewis, J. D. Chronic proton pump inhibitor therapy and the risk of colorectal cancer. Gastroenterology 133, 748-754 (2007).

27. Hwang, I. C., Chang, J. \& Park, S. M. Emerging hazard effects of proton pump inhibitor on the risk of colorectal cancer in low-risk populations: a Korean Nationwide Prospective Cohort Study. PLoS ONE 12, e0189114 (2017)
28. Kozuka, S., Nogaki, M., Ozeki, T. \& Masumori, S. Premalignancy of the mucosal polyp in the large intestine: II. Estimation of the periods required for malignant transformation of mucosal polyps. Dis. Colon Rectum 18, 494-500 (1975).

29. Belanger, C. F., Hennekens, C. H., Rosner, B. \& Speizer, F. E. The nurses' health study. Am. J. Nurs. 78, 1039-1040 (1978).

30. Colditz, G. A. \& Hankinson, S. E. The Nurses' Health Study: lifestyle and health among women. Nat. Rev. Cancer 5, 388-396 (2005).

31. Troy, L. M., Michels, K. B., Hunter, D. J., Spiegelman, D., Manson, J. E., Colditz, G. A. et al. Self-reported birthweight and history of having been breastfed among younger women: an assessment of validity. Int. J. Epidemiol. 25, 122-127 (1996).

32. Giovannucci, E., Ascherio, A., Rimm, E. B., Colditz, G. A., Stampfer, M. J. \& Willett, W. C. Physical activity, obesity, and risk for colon cancer and adenoma in men. Ann. Intern. Med. 122, 327-334 (1995).

33. Lee, D. H., Keum, N. \& Giovannucci, E. L. Colorectal cancer epidemiology in the Nurses' Health Study. Am. J. Public Health 106, 1599-1607 (2016).

34. DerSimonian, R. \& Laird, N. Meta-analysis in clinical trials. Control Clin. Trials 7, 177-188 (1986)

35. Bardou, M., Barkun, A. N. \& Martel, M. Obesity and colorectal cancer. Gut 62, 933-947 (2013).

36. Zauber, A. G., Winawer, S. J., O’Brien, M. J., Lansdorp-Vogelaar, I., van Ballegooijen, M., Hankey, B. F. et al. Colonoscopic polypectomy and long-term prevention of colorectal-cancer deaths. N. Engl. J. Med. 366, 687-696 (2012).

37. Giovannucci, E., Egan, K. M., Hunter, D. J., Stampfer, M. J., Colditz, G. A., Willett, W. C. et al. Aspirin and the risk of colorectal cancer in women. N. Engl. J. Med. 333 609-614 (1995).

38. Minalyan, A., Gabrielyan, L., Scott, D., Jacobs, J. \& Pisegna, J. R. The gastric and intestinal microbiome: role of proton pump inhibitors. Curr. Gastroenterol. Rep. 19, 42 (2017). 